\title{
A simple all-optical format transparent time and wavelength demultiplexing technique for WDM \& Orthogonal-TDM Nyquist channels
}

\author{
Mohammad Amin Shoaie, Armand Vedadi, Camille-Sophie Brès
}

(1) Photonic Systems Laboratory (PHOSL), STI-IEL, EPFL, CH-1015 Lausanne, Switzerland, amin.shoaie@epfl.ch

\begin{abstract}
A$ novel and simple non-coherent method to demultiplex WDM and orthogonal TDM Nyquist signal is presented. Using intensity modulators and filter, demultiplexing from a $3 \times 8 \times 10=240 \mathrm{~Gb} / \mathrm{s}$ signal down to $10 \mathrm{~Gb} / \mathrm{s}$ is demonstrated.
\end{abstract}

\section{Introduction}

The steady growth of data traffic combined with the requirement for economic viability necessitates new technologies to reach fibre network capacity limits. A promising technique is the use of sinc-shaped Nyquist pulses that enable multiplexing channels in the time domain with no inter-symbol interference (ISI) while exhibiting a rectangular spectrum that alleviates the need for guard-band [1]. The sinc pulse is indeed the basic building block in most theoretical papers that have estimated capacity limits [2], and intense efforts are being made to generate optical Nyquist pulses and transmitters beyond the limit of electronics [3-5]. ISI-free demultiplexing of orthogonal time division multiplexed (O-TDM) Nyquist pulses can be obtained by sampling at the exact channel pulse time location. However, in practice these methods are energy inefficient while complex and expensive electrical or optical apparatus are required to minimise the sampling window [6-7]. An alternative method consists in implementing the inner product between the incoming data and a train of Nyquist pulses aligned with the desired channel. This approach has been implemented using balanced detection [8]. It requires a local optical Nyquist pulse generator, as well as some digital signal processing after photodetection to minimize the phase error. In this work, we suggest to implement all-optically the inner product of the signal with a train of sinc functions at the receiver: multiplication is realized using intensity modulators (IM) driven by RF tones in a manner similar to [5] and integration is then approximated by optical filtering, which also enables the selection of the desired WDM channel.

\section{Principle}

A train of optical sinc pulses $S(t)$ generated from a flat rectangular comb can be written as: $S(t)=\frac{E_{D 0}}{N} \frac{\sin \left(\pi N f_{R} t\right)}{\sin \left(\pi f_{R} t\right)} e^{2 \pi j f_{0} t} \quad$ where $\quad f_{R}$ is the repetition rate, $N$ is the number of spectral lines, $E_{D 0}$ is the pulse peak amplitude and $f_{0}$ is the carrier frequency. After passing through the delay line, the chain of intensity modulators driven by specific RF tones with adjusted bias and amplitude voltages as in [5] and the tunable filter centred at $f_{0}$, the signal becomes:

$$
\begin{aligned}
E_{o}\left(t^{\prime}, \tau\right) & =\int_{-\infty}^{\infty} S(t-\tau) \frac{V_{C 0}}{N} \frac{\sin \left[\pi N f_{R} t\right]}{\sin \left(\pi f_{R} t\right)} h\left(t-t^{\prime}\right) d t \\
& \times e^{2 j \pi f_{0} t^{\prime}}
\end{aligned}
$$

where $\tau$ is the delay between the peaks of $S(t)$ and the phase-locked RF tones, $V_{C 0}$ is proportional to the amplitude voltages, and $h(t)$ is the impulse response of the optical filter. When $h(t)$ is a rectangular function of width $1 / f_{0}=T_{S}$, Eq. (1) corresponds to the inner product between two repeating sinc functions over one symbol length, with $E_{o}\left(t^{\prime}, 0\right)=V_{C 0} E_{D} / N^{2}$ and $E_{o}\left(t^{\prime}, k /\left(N f_{R}\right)\right)=0$ for all non-zero integer $k$. For an O-TDM channel consisting of $N$ sinc-shaped Nyquist symbol pulses, any channel can therefore be demultiplexed without interference from adjacent channels by aligning it with the sinc pulse carver using the delay line. Note that by adjusting the filter central wavelength, the desired WDM channel can also simultaneously be selected. The principle is sketched in Fig. 1.

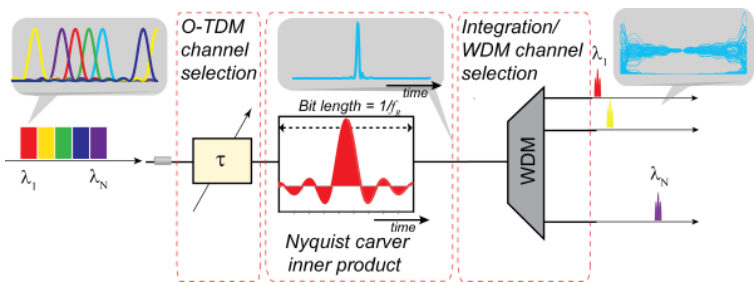

Fig. 1: Principle scheme of demultiplexing. The incoming sequence undergoes an inner product with Nyquist carver. A band-pass filter then integrates product and selects the WDM channels.

\section{Experimental Setup}

In order to validate the concept, an experiment was conducted using the setup depicted in Fig. 
2. To generate a Nyquist WDM and O-TDM signal, three tunable external cavity lasers (ECL) are wavelength multiplexed before being modulated by a $10 \mathrm{~Gb} / \mathrm{s}$ NRZ $2^{7}-1$ pseudorandom bit sequence (PRBS). A spool of $10 \mathrm{~km}$ long SMF is used to decorrelate the NRZ WDM channels. The method described in [5] is then implemented to perform high quality sincshaped Nyquist carving with $f_{1}=30 \mathrm{GHz}$ and $f_{2}=$ $10 \mathrm{GHz}$ on all WDM channels. An optical clock multiplier (OCM) is then used to produce 8 Nyquist uncorrelated O-TDM tributaries on each WDM channel for a total $240 \mathrm{~Gb} / \mathrm{s}$ aggregate rate. Careful tuning of the variable delay at each multiplication stage is required to ensure that the delay between two adjacent channels is equal to $1 /\left(N f_{R}\right)$ in order to obtain orthogonal multiplexing. To optimize noise figure of the generated signal, low-noise EDFA was used before the OCM.

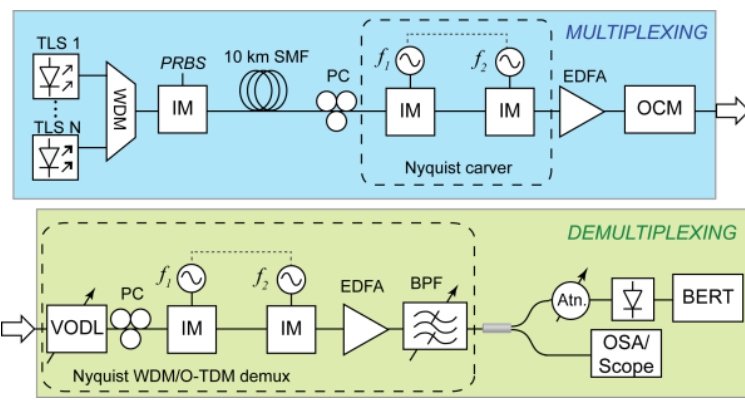

Fig. 2: TLS: tunable laser source, WDM: wavelength division multiplexer, IM: intensity modulator, PC: polarization controller, OCM: optical clock multiplier, VODL: variable optical delay line, BPF: band-pass filter, Atn: attenuator, BERT: bit-error rate tester.

For demultiplexing, a sinc-shape Nyquist carver similar to the one used for generating the O-TDM signal is made with two IMs driven at 10 $\mathrm{GHz}$ and $30 \mathrm{GHz}$, resulting in a close to perfect sinc-shaped Nyquist time window that carves the incoming Nyquist O-TDM sequence. A variable optical delay line (VODL) is placed before the demultiplexer to select the desired time tributary. The carved signal is finally amplified and passed through a Gaussian filter with $24 \mathrm{GHz}$ FWHM to emulate time integration of the optical field. Note that the filter acts as a WDM demultiplexer as well. The signal is acquired after a variable attenuator through a 10 $\mathrm{Gb} / \mathrm{s}$ digital photodetector. An oscilloscope and a bit error rate (BER) analyser is used to assess the demultiplexed signal quality.

\section{Results and discussion}

Fig. 3(a) shows the eye-diagram of Nyquist ODTM stream with 8 tributaries for a single WDM channel, acquired by a $500 \mathrm{GHz}$ optical sampling scope. The optical spectrum of the three WDM channels is also shown in Fig. 3(b). The central wavelength was chosen at $1539.9 \mathrm{~nm}$ while the two outer WDM channels were located at $\pm 90 \mathrm{GHz}$ from the central channel, thus realizing guard-band free WDM. The intensity modulators response varies negligibly over the $270 \mathrm{GHz}$ bandwidth, resulting in a simultaneous sinc-shaped Nyquist carving of all WDM combs.

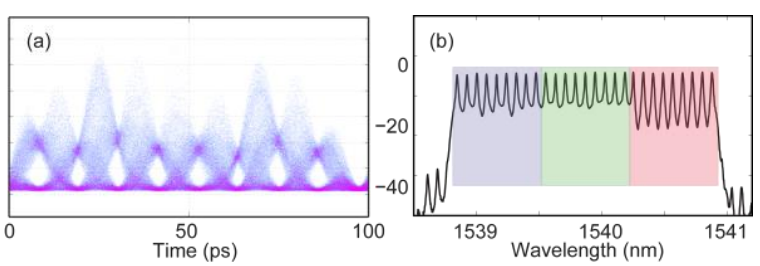

Fig. 3: (a) Eye-diagram of Nyquist O-TDM stream for single wavelength (b) Optical spectrum of 3 guard band-free WDM Nyquist channels.

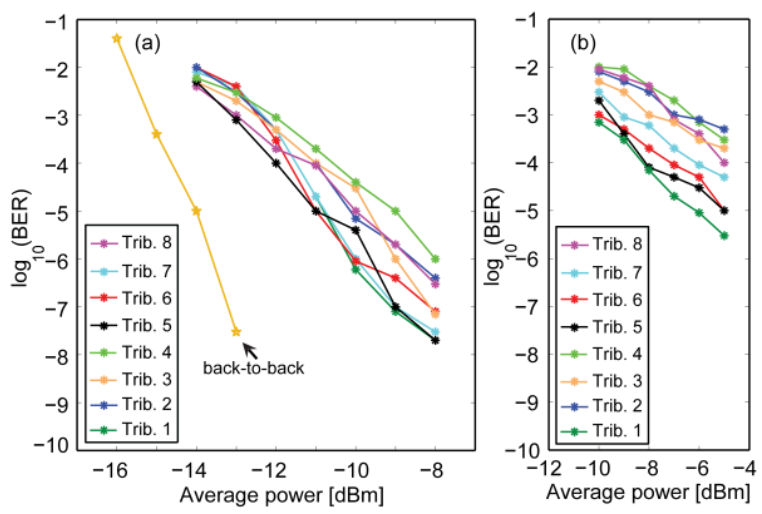

Fig. 4: Experimental BER of demultiplexed channels for (a) single wavelength (b) middle wavelength of three WDM signals

Fig. 4(a) depicts the BER of all 8 tributaries when only one WDM channel is present. On average, the performance for all tributaries is similar as a function of the received power. The variations observed are attributed to the laser phase noise that prevents perfect O-TDM in the OCM. Fig. 4(b) shows BER for all the 8 tributaries of the central WDM channel when all 3 WDM channels are on. It can be observed that performance is deteriorated in the case of WDM guard band free channels.

To have a better insight we performed a numerical simulation which accounts for the actual experimental conditions. Figure $5(\mathrm{a}-\mathrm{c})$ shows the simulated electrical eye-diagrams of a demultiplexed tributary for the case of one WDM channel (Fig. 5a) and 3 WDM channels (Fig. 5b). The eye diagram of an edge WDM channel is also shown in Fig. 5(c). The corresponding experimental eye-diagrams are shown in Fig. 5(d-f). The digital photodetector was modelled using a Butterworth electrical filter. The extracted $Q$-factors from the simulated results are in good agreement with BER measurements of Fig.3. Noise was not included in these simulations. Hence, we can infer that the $Q$ degradation is due to ISI between the O- 
TDM tributaries and crosstalk between the WDM channels.

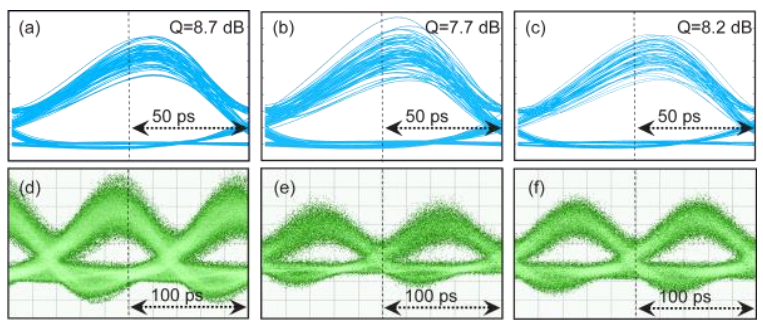

Fig. 5: Simulation and experimental demultiplexed eyediagram of a tributary in 8 Nyquist O-TDM for $(a, d)$ single wavelength, $(b, e)$ middle wavelength in 3 WDM channels, $(c, f)$ edge channel in 3 WDM channels.

Indeed, the impulse response of the filter used in the experiment is Gaussian rather than the required rectangular function. Numerical simulations were performed using a sinc-shaped filter in the frequency domain. Note that these filters could be made using fiber Bragg gratings [9]. Fig. 6(a) \& (b) show the optical eye diagram, after optical filtering with a Gaussian and sincshaped filter, respectively. As expected, a Gaussian impulse response filter leads to optical Gaussian symbol pulses of the demultiplexed channel, while the sinc-shaped filter leads to a NRZ stream. The optical Q-factor is enhanced in the case of sinc-shaped filtering, while some deterioration is still observed. The remaining deterioration is attributed to the fact that each tributary symbol pulse is located at the centre of its bit slot. As a result, the bit location of the OTDM tributaries do not coincide. Thus, when a tributary is demultiplexed, symbol switches from other tributaries lead to a non-zero ISI. Note that this effect is present in all demultiplexing schemes based on integration. To overcome this limitation, it was suggested to coincide the bit slots of all tributaries [10]. The difference between the former "bit non-assigned" and the latter "bit assigned" case is sketched in Fig. 6(c,d). Figure 6(c) shows that an infinite optical Q-factor is obtained when the bit-assigned arrangement is used. In practice however, the limited rise-fall time of NRZ modulators deteriorate the quality of the generated signal, in particular for the tributaries that are located at the edge of the bit slot. Figure 6(d) shows that for a rise/fall time of $10 \mathrm{ps}$, serious degradation of the optical Q-factor is observed for a tributary located at the edge of the assigned bit slot, whereas no noticeable degradation was observed in the bit non assigned case.
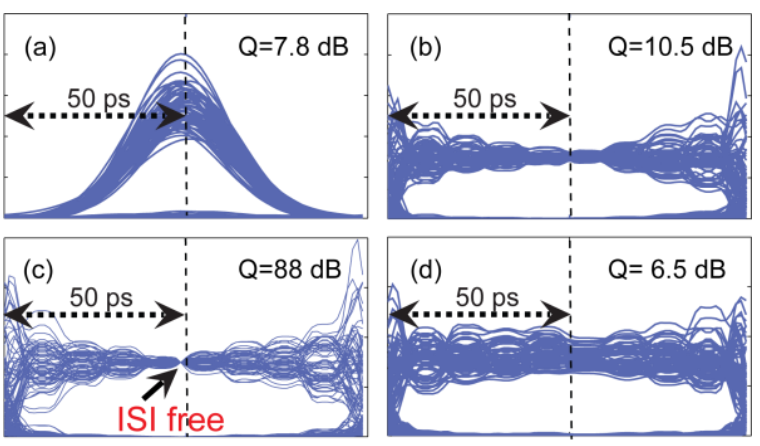

Fig. 6: Simulated optical demultiplexed eye-diagram of a tributary in 8 Nyquist O-TDM WDM channels for (a) Gaussian filter, bit non-assigned, (b) Nyquist filter, bit nonassigned, (e) Nyquist filter, bit assigned, (f) Nyquist filter, bitassigned including rise/fall time in a tributary at the edge of bit slot.

\section{Conclusions}

A simple and flexible method for simultaneous demultiplexing of WDM O-TDM sinc-shaped Nyquist stream based on the orthogonality of sinc pulses was implemented using intensity modulators, pulse positioning and filtering. Leveraging the ability to easily generate identical sinc gates at both transmitter and receiver, we demonstrated demultiplexing of $3 \times 8 \times 10=240 \mathrm{~Gb} / \mathrm{s}$ signal down to $10 \mathrm{~Gb} / \mathrm{s}$. The method does not need any coherent scheme, is format transparent and all-optical, hence compatible with WDM networks. Using sincshaped optical filters, the best performance achievable with demultiplexing schemes based on integration could be attained.

\section{References}

[1] D. Hillerkuss et al., "Single laser 32.5 Tbit/s Nyquist WDM transmission," Opt. Commun. Netw., Vol. 4, no. 10, p. 715 (2012).

[2] A. Mecozzi et al., "Nonlinear Shannon Limit in Pseudolinear Coherent Systems, " J. Lightwave Technol. Vol. 30, no.12, p. 2011 (2012).

[3] M. Nakazawa et al., "Ultrahigh-speed orthogonal TDM transmission with an optical Nyquist pulse train" Opt. Express, Vol. 20, no. 2, p. 1129 (2012)

[4] H. Hu et al., " $320 \mathrm{~Gb} / \mathrm{s}$ Nyquist OTDM received by polarization-insensitive time-domain OFT" Opt. Express, Vol. 22, no. 1, p. 110 (2014).

[5] M.A. Soto, et al, "Optical sinc-shaped Nyquist pulses of exceptional quality," Nature Communications 4, 2898 (2013).

[6] K. Harako et al. "Novel ultrafast TDM demultiplexing scheme using orthogonality in coherent Nyquist pulses, "ECOC, Cannes (2014)

[7] J. R. Stroud et al. "All-optical demultiplexing of Nyquist OTDM using a Nyquist gate," CLEO: Science and Innovations, SW1J-4, San Jose (2014).

[8] D. Kong et al. "A Novel Detection Scheme for Nyquist Optical Time-division Multiplexed Signal with Coherent Matched Sampling," OFC, W3C-4, Los Angeles (2015).

[9] A. Azana, "Ultrafast analog all-optical signal processors based on fiber-grating devices," IEEE Photonics, Vol. 2, no. 3, p. 359 (2010).

[10]A. Lowery et al., "All-optical generation of DFT-S-OFDM superchannels using periodic sinc pulses," Opt. Express, Vol. 22, no. 22, p. 27026 (2014). 